\title{
Ciclicidad en ovejas Santa Inês e Ideal en el subtrópico argentino
}

\author{
Arbues, R. ${ }^{1}$; F.Quintana, C. ${ }^{\text {; Yáñez, E. }}{ }^{2}$; Fernández, J. ${ }^{3}$ \\ ${ }^{1}$ Cátedra de Histología y Embriología, ${ }^{2}$ Cátedra de Nutrición y Alimentación, Fac.Cs.Veterinarias Univ.Nac.Nord. \\ (UNNE). ${ }^{3}$ Cátedra de Nutrición Animal, Fac.Cs.Agr.UNNE. Tel: 03794-421753 Corrientes (3400), Argentina. \\ E-mail: carofloresq@gmail.com
}

\begin{abstract}
Resumen
Arbues, R.; F.Quintana, C.; Yáñez, E.; Fernández, J.: Ciclicidad en ovejas Santa Inês e Ideal en el subtrópico argentino. Rev. vet. 29: 1, 3-8, 2018. Con el objetivo de caracterizar la ciclicidad anual de ovejas Santa Inês en ambiente subtropical, se desarrolló un ensayo en el departamento de Monte Caseros, Corrientes, Argentina. A 10 ovejas Santa Inês y 10 controles de raza Ideal se les tomaron muestras de sangre cada 10 días durante el otoño, invierno y primavera para determinar niveles séricos de progesterona y así clasificarlas en ovejas cíclicas o en anestro. Durante los primeros 20 días de ensayo recibieron suplementación alimentaria. El peso vivo (PV) se registró 2 veces en cada estación. Los datos se analizaron mediante tablas de frecuencia y prueba de chi cuadrado con grado de significancia de 0,5 . El $70 \%$ de las hembras Santa Inês presentó ciclicidad en otoño e invierno. En primavera este porcentaje descendió a $60 \%$. Las ovejas Ideal tuvieron un porcentaje de ciclicidad de 80,10 y $20 \%$ en otoño, invierno y primavera respectivamente. Se relacionó ausencia de ciclicidad con PV. Durante el período de suplementación alimentaria todas las hembras Santa Inês ganaron peso. Todas las ovejas que ciclaron en las tres estaciones estudiadas no tuvieron grandes variaciones de PV. Si bien hubo descenso, éste no fue suficiente para modificar la condición corporal. Entre las hembras que presentaron algún periodo de anestro se registraron descensos de peso mayores. Si bien la pérdida de peso pudo determinar el anestro, el tiempo de la señalización para el reinicio de la actividad estral podría ser variable. El análisis estadístico no arrojó diferencias significativas en ovejas Santa Inês pero sí lo hizo en ovejas Ideal. Se concluye que las ovejas tropicales, en ambientes subtropicales mantienen la ciclicidad anual y que la época del año no influye sobre la presentación de ciclos estrales en la raza Santa Inês.
\end{abstract}

Palabras clave: ovejas, progesterona, peso vivo, estacionalidad reproductiva, anestro.

\begin{abstract}
Arbues, R.; F.Quintana, C.; Yáñez, E.; Fernández, J.: Estrous cyclicity in Santa Inês and Ideal ewes in the argentine subtropics. Rev. vet. 29: 1, 3-8, 2018. Whit the aim of characterize the annual cyclicity of Santa Inês ewes in subtropical environment, the trial was developed in the department of Monte Caseros, Corrientes, Argentina. Ten Santa Inês ewes and ten controls Ideal race blood samples were taken every ten days during autumn, winter and spring to determine serum progesterone levels and classify them in cyclic ewes and anestrous ewes. During the first twenty days of trial they received food supplementation. Live weight was recorded twice in each season. Data were analyzed using frequency tables and chi-square test with significance level with 0.5 . The $70 \%$ of Santa Inês ewes presented estrous cycles in autumn and winter. This percentage fell to $60 \%$ in spring. Ideal ewes had a percentage of cyclicity 80,10 and 20 in autumn, winter and spring respectively. It was associated to the absence of cyclicity and live weight. During the period of food supplementation all Santa Inês females gained weight. All ewes that cyclized in the 3 seasons studied had no large variations in live weight. Although there was decrease in weight this was not enough to change the body condition. Among females who presented some period of anoestrus greater weight decreases they were recorded. Although weight loss could determine the anestro, time signaling for the resumption of estrous activity could be variable. Statistical analysis showed no significant differences in Santa Inês ewes but it did on Ideal ewes. In conclusion, tropical ewes in subtropicals environments maintain the annual cyclicity and the time of year does not influence the presentation of estrous cycles in the Santa Inês race.
\end{abstract}

Key words: ewes, progesterone, live weight, reproductive seasonality, anestrous. 


\section{INTRODUCCIÓN}

La estacionalidad de los ciclos sexuales, caracterizados por la existencia de un periodo de actividad sexual y otro de inactividad ovárica o anestro ${ }^{27}$ representa una adaptación natural de los ovinos para que las épocas de parto coincidan con las épocas de mejor clima y mayor disponibilidad de forrajes para una mejor tasa de supervivencia de la descendencia ${ }^{18}$.

Así, las hembras se vuelven sexualmente activas después del solsticio de verano, cuando los días comienzan a ser cada vez más cortos. Al contrario, la transición de días cortos a largos resulta en anestro ${ }^{35}$.

Sin embargo, esta estrategia se presenta mayormente en ovinos que viven en latitudes iguales o mayores a $35^{\circ}$, donde los cambios ambientales son contrastantes en las diferentes estaciones del año ${ }^{22}$. Las razas ovinas originarias de latitudes cercanas a los trópicos carecen de anestro estacional o los mismos son de menor duración ${ }^{27}$.

Santa Inês es una raza tropical, deslanada, originaria de Brasil. Entre sus características reproductivas se puede mencionar el poliestrismo anual en condiciones controladas de manejo y la alta frecuencia de partos múltiples, sobre todo dobles. Las hembras presentan partos aun con cría al pie, lo que posibilita intervalos entre partos de menor duración.

$\mathrm{Su}$ acentuada habilidad materna favorece la supervivencia perinatal ${ }^{5}$. Estos atributos la colocan en una posición estratégica como genética factible de ser usada en programas de mejoramiento por medio de selección o cruzamiento. La producción ovina de la región mesopotámica utiliza frecuentemente la raza Ideal, que produce lana de excelente calidad y tiene una destacada aptitud carnicera pero es poliéstrica estacional con un anestro breve ${ }^{12}$.

La obtención de un único parto en el año es el reflejo de una estación reproductiva estrecha que implica una oferta estacional de carne, leche, cuero y derivados. Esto favorece a que el consumo de carne ovina se restrinja solo a determinadas épocas del año. Por ello, resulta de sumo interés la evaluación de la actividad reproductiva de hembras ovinas de origen tropical, que verifique la ausencia de estacionalidad en otros puntos geográficos, para justificar su introducción en áreas productivas con mayor amplitud en los cambios de la longitud del día.

El objetivo del presente trabajo fue caracterizar la ciclicidad de las ovejas Santa Inês a lo largo del año en un ambiente subtropical.

\section{MATERIAL Y MÉTODOS}

El trabajo se desarrolló en el establecimiento agropecuario Don Donato, Colonia Mota, departamento de Monte Caseros, Corrientes. Su localización es $30^{\circ} 30^{\prime} \mathrm{S}$ y $58^{\circ} 02^{\prime} \mathrm{O}$, con clima subtropical húmedo y precipitaciones anuales de $1.100 \mathrm{~mm}$ promedio.

Se utilizaron ovejas sexualmente maduras no gestantes, las cuales conformaron dos grupos según su raza; el grupo 1 formado por 20 ovejas Santa Inês y el grupo 2 por 20 ovejas Ideal. Todas fueron mantenidas bajo las mismas condiciones de alimentación, sanidad y de manejo. Durante los primeros 20 días del ensayo las ovejas recibieron suplementación alimentaria a base de maíz picado y heno de alfalfa.

Estacionalidad reproductiva: los muestreos se distribuyeron en cuatro ventanas a lo largo del año: marzo-abril, junio-julio, septiembre-octubre y noviembrediciembre. Las muestras de sangre se tomaron a 10 ovejas de cada grupo, de la vena yugular, y se colectaron en tubos sin anticoagulante ${ }^{11}$. Inmediatamente se centrifugaron a $3000 \mathrm{rpm}$ por 10 minutos y se conservaron a $-20^{\circ} \mathrm{C}$ hasta el momento de su análisis.

El muestreo de marzo-abril (otoño) tuvo la finalidad de conocer el patrón de los niveles de progesterona en la época que las ovejas de la zona presentan ciclicidad y fertilidad mayores al resto del año. Las tres épocas de muestreo restantes se hicieron en los periodos del año (invierno-primavera) que las ovejas lanadas de la zona no presentan ciclicidad.

La determinación de los niveles de P4 se llevó a cabo en el Laboratorio de Reproducción Animal de la Facultad de Ciencias Veterinarias de la Universidad Nacional de La Plata. Cuando los niveles de la hormona fueron mayores a $0,5 \mathrm{ng} / \mathrm{ml}$ al menos una vez en la estación, se consideró que esa hembra era cíclica en esa estación.

Los datos del peso vivo se obtuvieron con balanza digital adaptada para ovinos, en todas las hembras en estudio, al iniciar el ensayo, luego a mitad del otoño, inicio, mediados y fin del invierno y al finalizar la primavera. Los datos de temperatura ambiente y precipitaciones fueron obtenidos del Servicio Meteorológico Nacional.

Las variables respuesta fueron del tipo cualitativas (ciclantes y no ciclantes). Los datos se analizaron mediante tablas de frecuencia y prueba de $\mathrm{Chi}^{2}$. El grado de significancia fue de 0,5 . El software utilizado para el análisis de datos fue Infostat $2002{ }^{19}$.

\section{RESULTADOS Y DISCUSIÓN}

Las frecuencias de hembras con actividad estral cíclica, Santa Inês e Ideal en cada estación estudiada, se presentan en la Figura 1.

Ciclicidad y peso vivo. Para un mejor análisis se subdividieron de la siguiente manera: Livianas $(31,8$ a $33,8 \mathrm{~kg}$ ), Intermedias (35 a 36,9 kg) y Pesadas (37,15 a $39,3 \mathrm{~kg}$ ). Los resultados de este análisis se presentan en la Figura 2.

Durante el período de suplementación alimentaria todas las hembras Santa Inês ganaron peso. Los porcentajes de pérdidas y/o aumentos de peso se presentan en la Tabla 1.

Una de las hembras del subgrupo Livianas cicló a partir de agosto, pudiendo vincularse la ausencia de ciclicidad con la pérdida del $13,7 \%$ del peso vivo (PV). Con la recuperación del peso se registró actividad cíclica en la misma. 
Tabla 1. Promedios de ganancia o pérdida de peso vivo en ovejas Santa Inês discriminados por período.

\begin{tabular}{lccc}
\hline estación & livianas & intermedias & pesadas \\
\hline promedio otoño & +3 & +3 & +3 \\
inicio invierno & $-10,5$ & -15 & -12 \\
promedio invierno & +1 & $+6,6$ & -5 \\
fin invierno & $+0,4$ & +8 & $+2,5$ \\
primavera & +16 & +10 & +16 \\
\hline
\end{tabular}

Valores en \%. Símbolos $+\mathrm{y}-$ delante del número indican si hubo ganancia o pérdida de peso corporal.

En el subgrupo Intermedias, si bien el PV se recuperó desde el inicio del invierno, en dos animales se registró anestro en diferentes estaciones, uno en invierno, en una hembra que perdió el $18 \%$ de su peso en el otoño. Aunque se fue recuperando, su ciclicidad se reinició recién en diciembre. El otro anestro fue en primavera, en una hembra que perdió $25 \%$ del $\mathrm{PV}$ en el otoño, pero aumentó $30 \%$ en el invierno. Si bien la pérdida de peso pudo determinar el anestro, el tiempo de la señalización para el reinicio de la actividad estral podría ser variable ${ }^{34}$.

Los mecanismos que relacionan el estado nutricional con las características de la secreción pulsátil de la hormona luteinizante (LH) no se conocen con exactitud. Se sabe que existen señales sanguíneas que reflejan el estado metabólico del animal y que pueden influir sobre la secreción de $\mathrm{LH}^{6,33}$. Es probable que los efectos inhibitorios de la baja nutrición sobre la secreción de LH involucren mecanismos del sistema nervioso central que controlan la secreción de gonadotropina (GnRH) por el hipotálamo ${ }^{15}$.

No obstante, se conoce poco acerca de cómo se informa el sistema nervioso central sobre su estado nutricional y de cómo esta información es traducida en una señal neuroendocrina. LH podría ser regulada por señales metabólicas presentes en la sangre, como ácidos grasos libres, insulina, tiroxina, hormona del crecimiento y factor de crecimiento insulínico-I (IGF-I) ${ }^{29}$.

Además, las reservas de grasa corporal regulan la secreción de LH a través de la hormona leptina. Probablemente, no sea una sola señal nutricional específica la

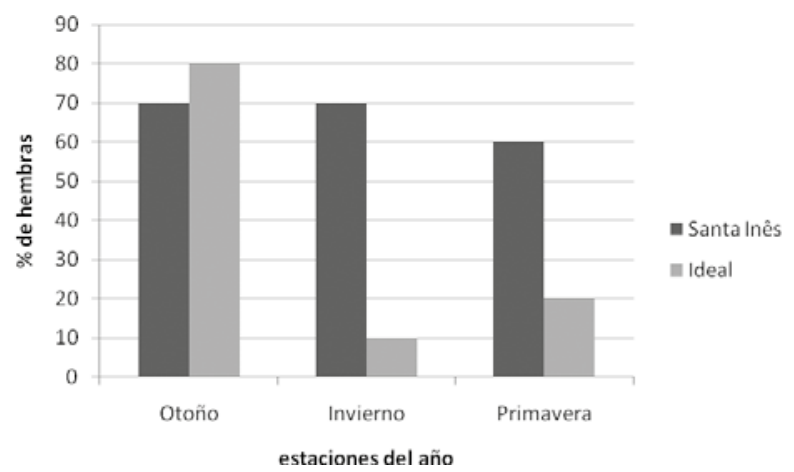

Figura 1. Porcentajes de ovejas cíclicas en las tres estaciones estudiadas. que controla la secreción de LH, sino más bien varios factores que actúan sinérgicamente en dicho proceso.

En el subgrupo de las ovejas Pesadas se detectó en un caso la ausencia de ciclicidad durante el invierno y disminución del $20 \% \mathrm{PV}$, que se reinició cuando fue recuperado la ciclicidad. No obstante, en otra hembra la ciclicidad permaneció cuando el PV disminuyó.

El PV de todas las ovejas que ciclaron en las tres estaciones estudiadas no tuvo grandes variaciones. $\mathrm{Si}$ bien hubo descenso de peso, no fue el necesario para modificar la condición corporal ${ }^{30}$. Los resultados del PV de las ovejas Ideal separadas en tres subgrupos se presentan en la en la Figura 3.

En las ovejas Ideal los cambios del PV fueron menos pronunciados. Las mismas iniciaron el ensayo con mayor PV, sin embargo lo perdieron hasta finalizar el invierno, incluso durante la suplementación. Esto posiblemente fue debido a la menor velocidad de ingestión comparada con la de las hembras tropicales, o a la mayor vulnerabilidad a las sucesivas extracciones de sangre.

En la mayoría de las especies se demostró que la reproducción no puede llevarse a cabo satisfactoriamente si la nutrición es deficiente ${ }^{34}$. La leptina, descubierta en 1994, sintetizada en los adipocitos, se presenta como la principal mediadora ${ }^{36}$. Esta hormona tiene receptores en hipotálamo, hipófisis, gónadas y tejido

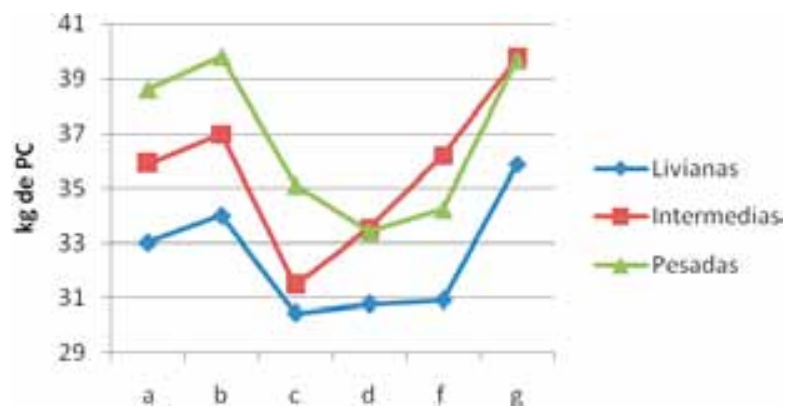

Figura 2. Peso vivo promedio de ovejas Santa Inês divididas en tres grupos, en diferentes momentos del estudio. a: inicio ensayo, b: promedio otoño, c: inicio invierno, d: promedio invierno, e: fin invierno, f: primavera.

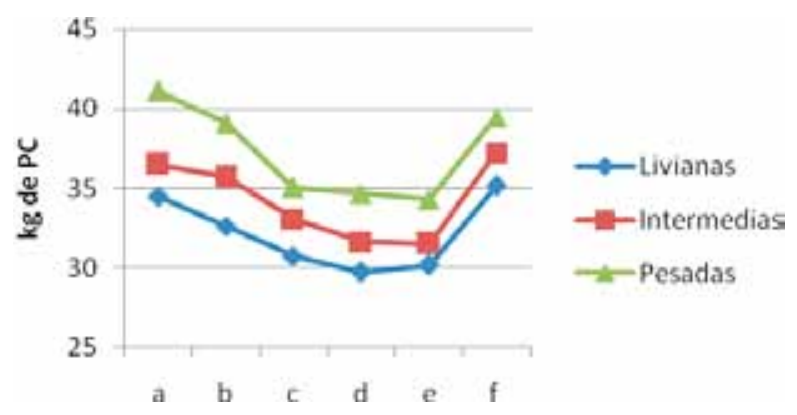

Figura 3. Peso vivo promedio de ovejas Ideal divididas en tres subgrupos, en diferentes momentos del estudio. a: inicio del ensayo, b: promedio otoño, c: inicio invierno, d: promedio invierno, e: fin invierno, f: primavera. 
Tabla 2. Promedios mensuales de temperaturas mínimas (TM), precipitaciones (P) y humedad relativa (HR) en Monte Caseros, Corrientes, año 2012.

\begin{tabular}{lcccccccccccc}
\hline & Ene & Feb & Mar & Abr & May & Jun & Jul & Ago & Sep & Oct & Nov & Dic \\
\hline TM $\left({ }^{\circ} \mathrm{C}\right)$ & 19,9 & 20,9 & 17,8 & 14,3 & 12,9 & 9,7 & 5,9 & 12,6 & 12,7 & 16,1 & 17,9 & 19,6 \\
$\mathrm{P}(\mathrm{mm})$ & 26,0 & 201,1 & 61,5 & 95,4 & 39,0 & 25,3 & 11,7 & 109,5 & 76,3 & 342,2 & 29,8 & 296,2 \\
HR $(\%)$ & 59 & 68 & 71 & 76 & 76 & 78 & 69 & 75 & 73 & 81 & 64 & 74 \\
\hline
\end{tabular}

adiposo ${ }^{14,32}$. Sus concentraciones en sangre pueden ser una señal metabólica a través de la cual el organismo regula la reproducción ${ }^{2,4,9,24,25,31}$.

Resultaría interesante conocer la velocidad con la cual trabaja este mediador informando cuando la hembra está en condiciones o no de reproducirse. En varios de los animales de este ensayo se observó que, en general, cuando hubo descenso de peso, la actividad cíclica cesó, pero esto sucedió en momentos diferentes.

También fue considerada la disminución de estros en los meses de primavera, en relación con temperatura y humedad ${ }^{13,17}$. Conociendo que Santa Inês es una raza rústica, adaptada a vivir en regiones donde las condiciones climáticas se caracterizan por temperaturas medias superiores a $18^{\circ} \mathrm{C}$, que oscilan poco y donde no se registran heladas independientemente que sea húmedo o árido, se podría esperar buena adaptación en una región subtropical a templada.

El efecto de las bajas temperaturas a las que estas ovejas están menos habituadas no fue detectado en la respuesta cíclica. Los datos de temperaturas mínimas medias, precipitaciones y humedad relativa ambiente se presentan en la Tabla 2.

Ciclicidad y fotoperíodo. El análisis estadístico de $\mathrm{Chi}^{2}$ para los datos de ciclicidad no arrojó diferencias significativas en ovejas Santa Inês, pero sí lo hizo en ovejas Ideal. Esto permite aceptar la hipótesis de que las ovejas tropicales, en ambientes subtropicales mantienen la ciclicidad anual, o en otras palabras, que la época del año no influye sobre la presentación de ciclos estrales en esta raza.

En Santa Inês existen antecedentes sobre la uniformidad en la distribución de sus ciclos estrales durante el año, a $21^{\circ} \mathrm{C}$ de latitud sur ${ }^{1}$. A diferencia del anterior, el presente trabajo aporta un dato más en este tema ya que se hizo a mayor latitud $\operatorname{sur}\left(30^{\circ} \mathrm{C}\right)$ y sin la presencia de machos. Este detalle no es menor, ya que la presencia del macho constituye una señal que promueve el desencadenamiento de la actividad reproductiva en las hembras ("efecto macho") ${ }^{3}$.

En un trabajo donde se realizó la caracterización de la actividad ovulatoria de ovejas Pelibuey y Suffolk a $19^{\circ}$ de latitud norte, con dieta de alta calidad y ausencia de carneros, muestra que el $60 \%$ de las hembras Pelibuey ovularon continuamente durante todo el estudio. Las ovejas Suffolk presentaron un anestro definido durante algunos meses de invierno y primavera en los dos años de estudio. Además, no se encontró correlación entre la temperatura ambiente y la actividad ovulatoria en ninguna de las dos razas.
El peso corporal de las ovejas Pelibuey no difirió significativamente en todo el ensayo. Las ovejas Suffo$1 \mathrm{k}$ aumentaron de peso de un año a otro, sin embargo esta variación no se correlacionó con la actividad ovulatoria de las hembras ${ }^{3}$. Entre las Pelibuey, algunas presentaron anestro en verano y otoño cuando las horas luz comienzan a decrecer. Es decir, no hubo sincronía entre el cese de la actividad ovulatoria y la señal que marca el ritmo endógeno de reproducción en ovejas.

En dos hembras Santa Inês se registró el mismo comportamiento. No presentaron actividad ovulatoria en otoño y comenzaron a ciclar en julio y agosto respectivamente, luego del solsticio de invierno. Frente a este hecho, se podría pensar en algún desacoplamiento entre el eje neuroendocrino-reproductivo y la influencia del fotoperíodo al exponer a las hembras a una información fotoperiódica diferente a la de su origen.

Sin embargo, la complejidad de las respuestas podría aumentar. En Black Belly el fotoperíodo templado simulado indujo marcada estacionalidad en la actividad ovulatoria. Llamativamente, ovejas expuestas a fotoperíodo tropical mostraron significativa estacionalidad en la actividad ovulatoria, diferente a lo que ocurre en la raza en condiciones naturales en su región de origen. Las señales del medio ambiente (temperatura, humedad, lluvias) actuarían en combinación con el fotoperíodo determinando la finalización o reactivación de la estación reproductiva ${ }^{8,16}$.

El presente ensayo muestra diferencias en la ciclicidad entre las razas estudiadas. Aunque algunas hembras Santa Inês presentaron un período de anestro, la mayoría mantuvo su capacidad de ciclar todo el año. Las ovejas Ideal detuvieron su actividad reproductiva en invierno y solo $20 \%$ cicló en primavera.

Algunos trabajos proponen la insensibilidad de las hembras al fotoperíodo, o bien insuficiente amplitud en los cambios del fotoperíodo para que los animales los perciban ${ }^{8}$. Podrían existir animales de bajo umbral de sensibilidad a los sutiles cambios del fotoperíodo tropical y que -por lo tanto- presenten anestro estacional normal de un año a otro.

El segundo tipo, cuya proporción puede llegar a $60 \%$, es el que presenta umbral de sensibilidad significativo a las variaciones de la longitud del día. Por lo tanto, estas ovejas son insensibles a los cambios de luz típicos de regiones tropicales, y continúan presentando ciclos estrales durante todo el año bajo estas condiciones. El tercer tipo de ovejas, con proporcionalidad de $20 \%$, tienen un umbral extremadamente alto, por lo cual se vuelven insensibles a los cambios en el fotoperíodo. Estas hembras continúan presentando ciclos 
estrales durante todo el año incluso bajo fotoperíodo de latitudes mayores $\left(56^{\circ}\right)^{3,26}$.

En el presente estudio, el $40 \%$ de ovejas Santa Inês presentó un período de anestro coincidente con la época en que los días comienzan a alargarse, pero este período fue más corto que el de las ovejas lanadas. A su vez existió alto porcentaje de hembras que continuó ciclando luego del solsticio de invierno, soportando la idea de que las ovejas tropicales tengan diferentes umbrales de sensibilidad a los cambios del fotoperíodo.

Una posible causa de estas variaciones es la existencia de un efecto enmascarador $y / 0$ amortiguador del ovario, que permite que los ciclos ováricos sigan ocurriendo a pesar de las variaciones en los niveles de gonadotrofinas ${ }^{20,21}$. Otra posible causa de variabilidad puede radicar en la secreción de melatonina, hormona secretada durante las horas de oscuridad ${ }^{10,23,28}$.

Los niveles plasmáticos nocturnos de melatonina son altamente variables entre individuos, pero repetibles para un mismo individuo, especialmente en ovejas y en humanos ${ }^{7}$. En este caso, la variabilidad de las hembras no estaría dada por cuan sensibles sean al fotoperíodo, sino por las concentraciones de melatonina que éstas alcanzan.

En ovejas que ciclaron continuamente bajo fotoperíodo $12 \mathrm{~h} / \mathrm{luz}-12 \mathrm{~h} /$ oscuridad, se observó que en algunas de ellas la elevación nocturna de melatonina fue pequeña, mientras que en otras fue marcada ${ }^{20}$. De acuerdo a este trabajo parece más probable que la variabilidad en la respuesta a un fotoperíodo diferente no se debería a diferencias en la secreción de melatonina sino más a bien a variaciones relacionadas a la forma en que se procesa la señal de la misma en el sistema nervioso central.

Las ovejas de raza Santa Inês mantuvieron la capacidad de presentar ciclos estrales durante todo el año en un ambiente subtropical. Esto demuestra que es factible la introducción de la raza tropical ya que controlando las condiciones de manejo, se podrá aprovechar su característica de "poliestrica continua" y de este modo conseguir desestacionalizar en primera instancia, la época de partos y consecuentemente la oferta de carne ovina. Por otra parte, la observación de períodos de anestro en algunas hembras tropicales, hace necesario seguir investigando cuáles fueron las causas. Las ovejas de raza Ideal demostraron, como era de esperar, una marcada época de anestro.

Agradecimientos. Al Servicio Meteorológico Nacional por proporcionar los datos de temperaturas, precipitaciones y humedad. A la Sra. Mabel Yáñez, propietaria del establecimiento Don Donato, por su valioso apoyo y colaboración en el desarrollo de los trabajos.

\section{REFERENCIAS}

1. Almeida P. et al. 2007. Annual characteristics of estrous activity in wool and hair ewe lambs under subtropical conditions. Sci Agric (Piracicaba Br.), 64: 468-475.
2. Amstalden M. et al. 2000. Leptin gene expression, circulating leptin, and luteinizing hormone pulsatility are acutely responsive to short-term fasting in prepuberal heifers: relationships to circulating insulin and insulinlike growth factor. Biol Reprod 63: 127-133.

3. Arroyo L, Gallegos J, Villa A, Berruecos J, Perera G, Valencia J. 2007. Reproductive activity of Pelibuey and Suffolk ewes at $19^{\circ}$ north latitude. Anim Reprod Sci 102: 24-30.

4. Barash I, Cheung C, Weigle D. 1996. Leptin is a metabolic signal to the reproductive system. Endocrinology 173: 3144-3147.

5. Bueno M, Da Cunha E, Dos Santos L, Veríssimo C. 2006. Uma boa alternativa para a produçao intensiva de carne de cordeiros na regiao Sudeste. http://www.infobibos.com/Artigos/2006_2/SantaInes/index.htm

6. Butler W. 2000. Nutritional interactions with reproductive performance in dairy cattle. Anim Reprod Sci 60: 449-457.

7. Chemineau P, Beltran I, Daveau A, Bodin L. 1996. Hight repeatability of the amplitude and duration of the nycthemeral rhythm of the plasma melatonin concentrations in the Ile de France ewes. J Pineal Res 21: 1-6.

8. Chemineau P, Daveau A, Cognié Y, Aumont G, Chesneau D. 2004. Seasonal ovulatory activity exists in tropical Creocle female goats and Black Belly ewes sujested to a temperate photoperiod. BMC Phisiology 4: 12-23.

9. Cheung C, Thornton J, Kuijper J, Weigle D, Clifton D, Steiner R. 1997. Leptin is a metabolic gate for the onset of puberty in the female rat. Endocrinology 138: 855-858.

10. Dardente H, Birnie M, Lincoln G, Hazlerigg D. 2008. $\mathrm{RF}$ amide-related peptide and its cognate receptor in the sheep: cDNA cloning, mRNA distribution in the hypothalamus and the effect of photoperiod. J Neuroendocrinol 20: 1252-1259.

11. Delpino A, González SC. 1993. Evaluación del comportamiento reproductivo en pequeños rumiantes tropicales utilizando los perfiles de progesterona. Revista Cientifica FCV-LUZ 3: 231-247.

12. De Gea G. 2007. El ganado lanar en Argentina, $2^{\mathrm{a}}$ Ed., Edit. Universidad Nacional de Río Cuarto, Córdoba, Argentina, $279 \mathrm{p}$.

13. De la Isla G, Ayala A, Aké J, González A. 2010. Efecto de la condición corporal y la época del año sobre el ciclo estral, estro, desarrollo folicular y tasa ovulatoria en ovejas Pelibuey mantenidas en condiciones de trópico. Vet Méx 41: 167-175.

14. Dyer C, Simmons J, Matteri R, Keisler D. 1997 Effects of an intravenous injection of NPY on leptin and NPYY1 receptor mRNA expression in ovine adipose tissue. Domest Anim Endocrinol 14: 325-333.

15. Ebling F, Wood R, Karsch F, Vannerson L, Suittie J, Bucholtz D, Schall R, Foster O. 1990. Metabolic interphases between growth and reproduction III. Central mechanisms controlling pulsatile luteinizing hormone secretion in the nutrionally growth-restricted female lambs. Endocrinology 126: 2719-2727.

16. Gómez A, Malpaux B, Daveau A, Taragnat C, Chemineau P. 2008. Genetic variability in melatonin secretion originates in the number of pinealocytes in sheep. J Endocrinol 172: 397-404. 
17. González A, Murphy B, Foote W, Ortega E. 1992. Circannual estrous variations and ovulation rate in Pelibuey ewes. Small Ruminant Res 8: 225-232.

18. Horta A, Gonçalves S. 2006. Bioestimulaçao pelo efeito macho na indução e sincronização da actividade ovárica em pequenos ruminantes. Anais XVI Congresso de Zootecnia, Esc. Sup. Agrária de Castelo Branco, Portugal, p. 95-108.

19. Infostat 2002. Infostat estudiantil, version 2.0. Grupo Infostat/FCA, Universidad Nacional de Cordoba. Ed. Brujas, Córdoba, Argentina.

20. Jackson G, Jansen H, Kao C. 1990. Continuous exposure of Suffolk ewes to an equatorial photoperiod disrupts expression of the annual breeding season. Biol Reprod 42: 63-73.

21. Jansen H, Jackson G. 1993. Circannual rhythms in the ewe: patterns of ovarian cycles and prolactin secretion under two different constant photoperiods. Biol Reprod 49: 627-634.

22. Lindsay D. 1991. Reproduction in the sheep and goat. In: Reproduction in domestic animals (Cupps PT edit.), Academics Press, California-USA, $294 \mathrm{p}$.

23. Morgan P, Hazlerigg D. 2008. Photoperiodic signaling through the melatonin receptor turns full circle. J Neuroendocrinol 20: 820-826.

24. Mostyn A, Keisler R, Weeb T, Stephenson A, Symonds M. 2001. The role of leptin in the transition from fetus to neonate. Proc Nutr Soc 60: 187-194.

25. Nagatani S, Zeng Y, Keisler D, Foster D, Jaffe C. 2000. Leptin regulates pulsatile luteinizing hormone and growth hormone secretion in the sheep. Endocrinology 141: 39653975 .
26. Porras A. 1999. Efectos del fotoperíodo artificial sobre la actividad reproductiva de la oveja Pelibuey. Tesis de Doctorado en Ciencias Veterinarias, Fac. Vet.\& Zoot., Univ. Nac. Autónoma de México, México.

27. Porras A, Zarco L, Valencia J. 2003. Estacionalidad reproductiva en ovejas. Ciencia Veterinaria 9: 2-25.

28. Reiter R, Tan D, Manchester L, Paredes S, Mayo J, Sainz R. 2009. Melatonin and reproduction revisited. Biol Reprod 81: 445-456.

29. Roche J, Mackey D, Diskin M. 2000. Reproductive management of pospartum cows. Anim Reprod Sci 60: 703-712.

30. Sánchez F. 2003. Condición corporal en ovejas. www. producción-animal.com.ar.

31. Sansinanea A, Cerone S, Zonco I, García C, Auza N. 2001. Serum leptin levels in cattle with different nutritional conditions. Nutrition Res 21: 1045-1052.

32. Spicer L, Francisco C. 1997. The adipose obese gene product, leptin: evidence of a direct inhibitory role in ovarian function. Endocrinology 138: 3374-3379.

33. Steiner R, Cameron J, McNeill T, Clifton D, Bremer W. 1983. Metabolic signals for the onset of puberty In: Neuroendocrine Aspects of Reproduction (Norman RL, editor), Academic Press, London, p. 183-227.

34. Velasco H, Gutiérrez C. 2008. Leptina: interacción nutrición-reproducción. Quehacer Cientifico en Chiapas 1: 645-652.

35. Yeates N. 1949. The breeding season of the sheep with particular reference to its modification by artificial means using light. J Agric Sci 39: 1-43.

36. Zhang Y, Proenca R, Maffei M, Barone M, Leopold L, Friedman J. 1994. Positional cloning of the mouse obese gene and its human homologue. Nature 372: 425-432. 